\title{
Gerhard Jackisch
}

Mikrovariabilität und Zustandsgrößen von hellen Sternen

Veröffentlichungen der Sternwarte in Sonneberg - Band 5 . Heft 5 

DEUTSCHE AKADEMIE DER WISSENSCHAFTEN ZU BERLIN

\section{Veröffentlichungen der Sternwarte in Sonneberg}

Band $5 \cdot$ Heft 5

GERHARD JACK ISCH

\section{Mikrovariabilität und Zustandsgrößen von hellen Sternen}

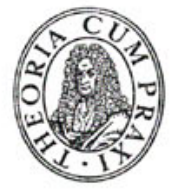

Akademie-Verlag Berlin 1963 
Erschienen im Akademie-Veriag GmbH, Berlin W 8, Leipziger Straße 3-4 Copyright 1963 by Akademie-Verlag $\mathrm{GmbH}$ Lizenznummer: $202 \cdot 100 / 495 / 63$

Gesamtherstellung: VEB Druckerei „Thomas Müntzer" Bad Langensalza Bestell-Nr.: 2006/V/5 - ES 18 D 4 - Preis: DM 15,- 